PROCEEDINGS OF THE

AMERICAN MATHEMATICAL SOCIETY

Volume 134, Number 1, Pages 295-301

S 0002-9939(05)07935-9

Article electronically published on June 3, 2005

\title{
EVERY REIDEMEISTER MOVE IS NEEDED FOR EACH KNOT TYPE
}

\author{
TOBIAS J. HAGGE
}

(Communicated by Ronald A. Fintushel)

\begin{abstract}
We show that every knot type admits a pair of diagrams that cannot be made identical without using Reidemeister $\Omega_{2}$-moves. The proof is compatible with known results for the other move types, in the sense that every knot type admits a pair of diagrams that cannot be made identical without using all of the move types.
\end{abstract}

\section{INTRODUCTION}

Reidemeister proved [3] that given two diagrams of ambient isotopic links, there is a sequence of transformations on one of the diagrams that gives an explicit isotopy. Each transformation is either a planar isotopy, a cusp move (class $\Omega_{1}$ ), a self-tangency move (class $\Omega_{2}$ ), or a triple point move (class $\Omega_{3}$ ). Every introductory knot theory textbook describes these move types; alternatively, see [2].

Two diagrams are equivalent if such a sequence exists, and the sequence is a Reidemeister sequence for the pair. Sometimes a Reidemeister sequence is specified without mention of the second diagram; in this case the second diagram is the result of applying the moves in the sequence. If, for a given diagram pair and $n \in\{1,2,3\}$, there is a Reidemeister sequence for the pair that does not contain an $\Omega_{n}$-move, the pair is $\Omega_{n}$-independent. Otherwise the pair is $\Omega_{n}$-dependent.

Since $\Omega_{1}$-moves are the only moves that change the winding number of a diagram, it is clear that every link type admits $\Omega_{1}$-dependent diagram pairs. Olof-Petter Östlund 2 has shown that every link type admits $\Omega_{3}$-dependent diagram pairs, as well as pairs that are simultaneously $\Omega_{1}$-dependent and $\Omega_{3}$-dependent. In the case of links with at least two components, $\Omega_{2}$-moves are the only moves that change the number of intersections between components. Thus such links admit $\Omega_{2}$-dependent diagram pairs. Vassily Manturov [1] has recently shown that a connected sum of any four distinct prime knots admits $\Omega_{2}$-dependent diagram pairs. We consider $\Omega_{2}$ moves in more generality, and construct $\Omega_{2}$-dependent diagram pairs for every knot type. Our approach is similar to Manturov's, but was developed independently. We conclude by showing that for each knot type one can construct a diagram pair that is simultaneously $\Omega_{1}$-dependent, $\Omega_{2}$-dependent, and $\Omega_{3}$-dependent.

Received by the editors May 20, 2004 and, in revised form, August 18, 2004.

2000 Mathematics Subject Classification. Primary 57M25.

The author thanks Charles Livingston, Zhenghan Wang, Scott Baldridge, and Noah Salvaterra for their helpful comments, and Vladimir Chernov for pointing out this problem.

(C)2005 American Mathematical Society Reverts to public domain 28 years from publication 


\section{MAIN THEOREM}

Briefly, the structure of the main argument is as follows. We give conditions on a knot diagram that severely limit what one can accomplish without $\Omega_{2}$-moves. The only transformations possible amount to replacing the edges in the original diagram with unknotted $(1,1)$-tangles (by a $(1,1)$-tangle we mean a single stranded tangle). We show that there is a diagram resulting from a single $\Omega_{2}$-move that cannot be attained without $\Omega_{2}$-moves. The result is then generalized slightly to construct $\Omega_{2^{-}}$ dependent diagram pairs for every knot type. Finally, taking pairwise connected sums with diagrams from Östlund's argument gives a pair of diagrams for each knot type that is simultaneously $\Omega_{1}$-dependent, $\Omega_{2}$-dependent, and $\Omega_{3}$-dependent.

Let $D$ be a planar knot diagram in general position. A polygon $p$ in $D$ is the boundary of a connected component of the complement $D^{c}$ of $D$ in the plane. Call $p$ a 0 -gon if $D$ contains no crossing points. Call $p$ an $n$-gon if, when all crossing points of $D$ that lie on $p$ are removed, the remainder consists of $n$ connected components homeomorphic to an open interval. Call the points so removed the vertices of $p$, and the connected components the edges. Note that $p$ can have fewer vertices than edges, and a 0 -gon does not have any edges.

Theorem 1. Let $D$ be a diagram for which the following hold:

(1) There are no 0-gons, 1-gons, or 2-gons,

(2) The first $\Omega_{n}$-move cannot be an $\Omega_{3}$-move.

Then any Reidemeister sequence not containing an $\Omega_{2}$-move does nothing more up to planar isotopy than replace the edges of $D$ with unknotted $(1,1)$-tangles. Furthermore, one can apply a single $\Omega_{2}$-move that crosses two distinct edges of $D$, giving a diagram $D^{\prime}$ such that the pair $\left\{D, D^{\prime}\right\}$ is $\Omega_{2}$-dependent.

Figure 1 satisfies the above preconditions. Figure2 gives an unknotted example. There are other examples, such as alternating diagrams with no 0-gons, 1-gons, or 2 -gons.

Proof. Suppose that $\Omega_{2}$-moves are disallowed. Let $\left\{k_{i}\right\}$ be the set of crossing points in $D$. In order for one of the $k_{i}$ to take part in an $\Omega_{1}$-move (in the sense that $k_{i}$ appears in at least one of the pictures that locally describe the move), it must be the vertex of a 1-gon. In order for a $k_{i}$ to take part in an $\Omega_{3}$-move, it must be one

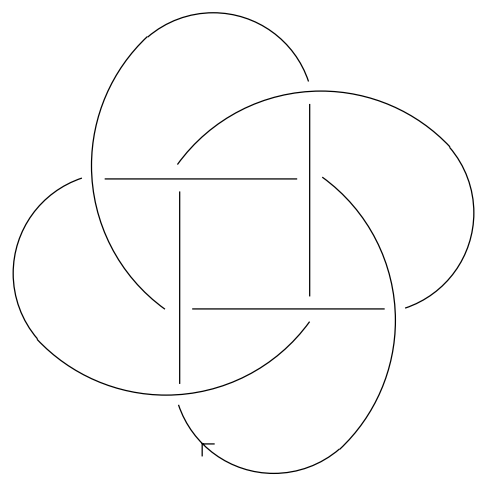

Figure 1. A simple diagram satisfying the preconditions of Theorem 1 


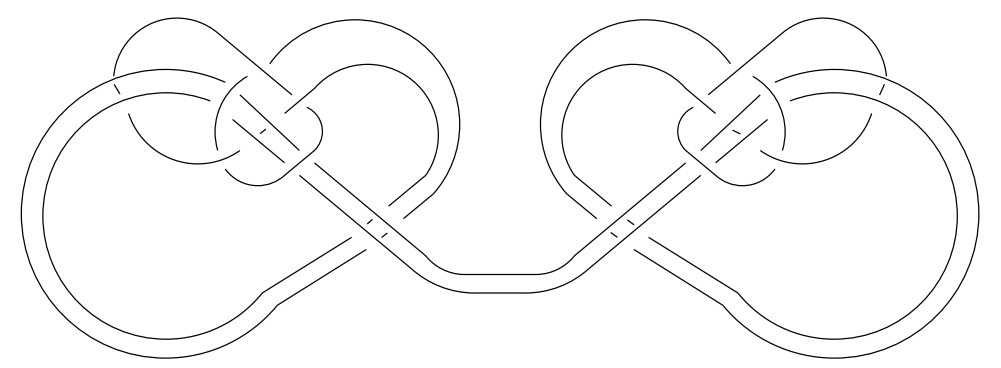

Figure 2. A diagram of the unknot satisfying the preconditions of Theorem 1.

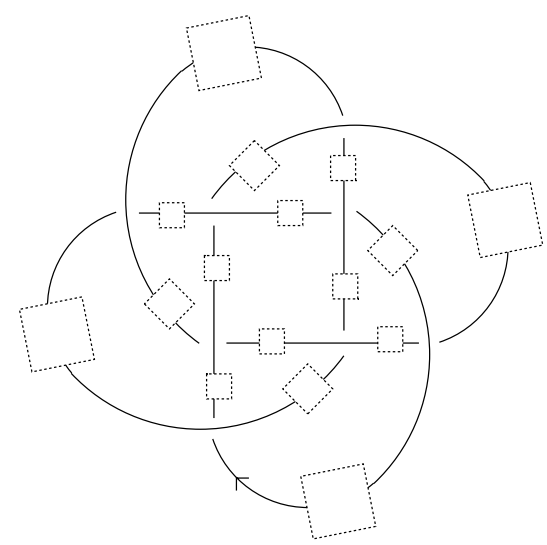

Figure 3. Figure 1, with edges replaced by unknotted $(1,1)$ tangles. Each dotted box represents an unknotted $(1,1)$-tangle.

of the vertices of a 3 -gon. Since $D$ contains no 1-gons, and none of its 3-gons admit an $\Omega_{3}$-move, no $k_{i}$ can take part in the first $\Omega_{n}$-move.

Suppose that a sequence of moves has been made such that no $k_{i}$ has taken part in an $\Omega_{1}$-move or an $\Omega_{3}$-move. Then the diagram is isotopic to the original diagram with each of the edges replaced by an unknotted $(1,1)$-tangle. (Figure 3 provides an illustration, where each dotted box contains a $(1,1)$-tangle.) The $(1,1)$-tangles cannot intersect because any $\Omega_{1}$-move in which no $k_{i}$ takes part is just a kink on a single $(1,1)$-tangle, and any $\Omega_{3}$-move in which no $k_{i}$ takes part cannot cause the intersection of two $(1,1)$-tangles that did not intersect before the move.

Now, it is still impossible for one of the $k_{i}$ to take part in an $\Omega_{1}$-move or an $\Omega_{3}$-move. For, given a $k_{i}$, every polygon $p$ that contains $k_{i}$ as a vertex looks like one of the polygons in $D$ that contain $k_{i}$, possibly with some extra edges due to the $(1,1)$-tangles. Thus, $p$ has at least three edges, and $p$ has exactly three edges only when it is one of the 3-gons in $D$ (here it is necessary that there are no 2-gons in $D$ ). Since none of the 3 -gons in $D$ admit $\Omega_{3}$-moves, no $k_{i}$ can take part in an $\Omega_{1}$-move or an $\Omega_{3}$-move.

Thus, up to isotopy, if $\Omega_{2}$-moves are not allowed, then a sequence of moves on $D$ will fix the $k_{i}$ and replace the edges of $D$ with unknotted $(1,1)$-tangles. This gives the first part of the theorem. 


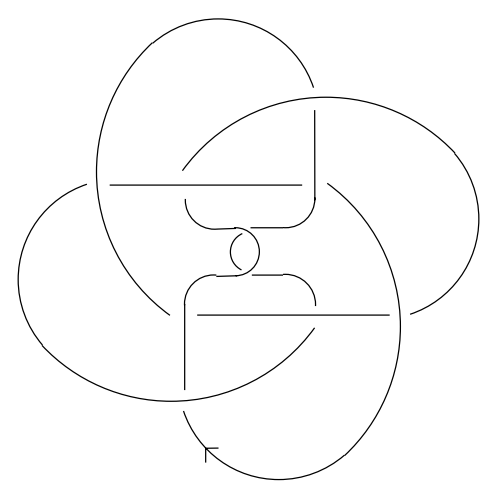

FIgURE 4. This diagram cannot be derived from Figure 1 without an $\Omega_{2}$-move.

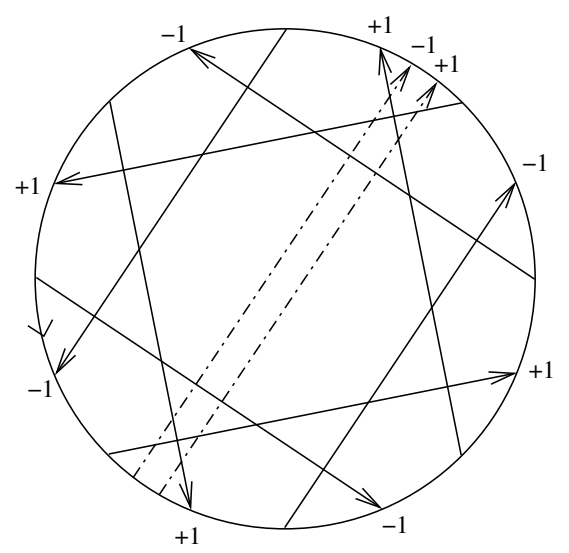

Figure 5. The Gauss diagram for Figure 4, Removing the dashed arrows gives the Gauss diagram for Figure 1

For the second part of the theorem, let the diagram $D^{\prime}$ be created by performing a single $\Omega_{2}$-move that crosses two distinct edges of $D$. Figure 4 provides an example. Let $E$ be a diagram such that $(D, E)$ is an $\Omega_{2}$-independent diagram pair. There are easy ways to show that $D^{\prime}$ and $E$ cannot be isotopic. For instance, one could show that if $D^{\prime}$ and $E$ have the same number of crossings, $E$ must contain a 1gon, but $D^{\prime}$ contains no 1-gons. The following argument is more complicated but straightforwardly generalizes to the full result.

Every oriented knot diagram $B$ is given by a smooth immersion $\phi$ from the oriented circle $S^{1}$ to the plane $\mathbb{R}^{2}$ with decorated crossing points. This immersion is unique up to orientation-preserving self-diffeomorphisms of $S^{1}$. The map $\phi$ is one to one except at crossing points, where it is two to one. The Gauss diagram $G_{B}$ for $B$ is constructed from $S^{1}$ by drawing a signed arrow between the two elements of $\phi^{-1}(k)$ for each crossing point $k$ of $B$. Each arrow points toward the under strand of the crossing. The sign of each arrow is the sign of that crossing, either +1 or -1 according to the standard convention. Figures 5 and 7 give examples. For a slightly lengthier introduction to Gauss diagrams, see [2]. 
Let $D$ be given an orientation. This gives an orientation for $E$. Consider the Gauss diagrams $G_{D}$ and $G_{E}$ of $D$ and $E$. One can see that $G_{E}$ is just $G_{D}$ with some extra arrows representing the $(1,1)$-tangles. It is easy to verify that none of the extra arrows intersect the arrows of $G_{D}$.

The $\Omega_{2}$-move that takes $D$ to $D^{\prime}$ adds two arrows $a_{1}$ and $a_{2}$ to $G_{D}$, giving the Gauss diagram $G_{D^{\prime}}$. The heads of $a_{1}$ and $a_{2}$ are adjacent on $S^{1}$, as are the tails. Thus, any arrow that intersects $a_{1}$ or $a_{2}$ must intersect both $a_{1}$ and $a_{2}$. One can easily show that both $a_{1}$ and $a_{2}$ must intersect at least one of the arrows in $G_{D}$.

Any Gauss diagram containing a pair of arrows with adjacent heads and tails represents a knot diagram that contains a 2-gon. Since $D$ contains no 2-gons, any copy of $G_{D}$ in $G_{D^{\prime}}$ intersecting no other arrows cannot contain $a_{1}$ and $a_{2}$, and so contains neither $a_{1}$ nor $a_{2}$. Thus the addition of the arrows $a_{1}$ and $a_{2}$ causes $G_{D}$ to intersect other arrows without creating any new copies of $G_{D}$ that do not intersect other arrows. This reduces the number of copies of $G_{D}$ intersecting no other arrows from one to zero. Since $E$ has at least one copy of $G_{D}$ intersecting no other arrows, $D^{\prime}$ and $E$ are not isotopic. This proves the theorem.

Theorem 2. Every knot type admits an $\Omega_{2}$-dependent diagram pair.

Proof. Figure 2 is a diagram of the unknot and so Theorem 1 gives an $\Omega_{2}$-dependent unknotted diagram pair. Removing a small closed line segment from one of the edges of a diagram $A$ results in a $(1,1)$-tangle $T$ having the same knot type as $A$. If $B$ is another knot diagram, then $A \# B$ is obtained by replacing an edge in $B$ with a $(1,1)$-tangle planar isotopic to $T$.

Let $D$ be the diagram in Figure 2, Let $F$ be a diagram given by replacing one or more of the edges of $D$ with arbitrary $(1,1)$-tangles. Then by the same argument as in Theorem 1 move sequences not containing $\Omega_{2}$-moves can only replace these $(1,1)$-tangles with other $(1,1)$-tangles of the same knot types. Now, $G_{F}$ must contain at least one copy of $G_{D}$ that intersects no other arrows. It may contain more (for instance if $F=D \# D$ ). However, none of these copies can be altered or intersected with other arrows by a Reidemeister sequence not containing $\Omega_{2}$-moves. Let $F^{\prime}$ be the result of a single $\Omega_{2}$-move that crosses two distinct edges of $F$. Then the created arrows on $G_{F^{\prime}}$ intersect a copy of $G_{D}$ that intersected nothing else in $G_{F}$. This reduces the number of copies of $G_{D}$ that intersect no other arrows, just as in Theorem 11. Thus $\left(F, F^{\prime}\right)$ is an $\Omega_{2}$-dependent diagram pair.

We wish to construct diagram pairs that are simultaneously $\Omega_{1}$-dependent, $\Omega_{2}$ dependent, and $\Omega_{3}$-dependent. In order to do this we briefly summarize a portion of the proof given by Östlund in [2], enough to prove the existence of $\Omega_{3}$-dependent diagram pairs. The reader should see [2] for details.

Theorem 3. Every knot type admits an $\Omega_{1}$-dependent, $\Omega_{2}$-dependent, $\Omega_{3}$-dependent diagram pair.

Proof. Östlund's proof counts the signed number of instances of the Gauss subdiagram given in Figure 6. The sign of each subdiagram is given by the product of the signs of the crossings in the subdiagram. Östlund shows that this count is invariant under $\Omega_{1}$-moves and $\Omega_{2}$-moves, but can vary under $\Omega_{3}$-moves. Östlund uses this count to prove that for every knot type there is a diagram pair that is simultaneously $\Omega_{1}$-dependent and $\Omega_{3}$-dependent. As an example, the Gauss diagram in Figure 7 represents a figure eight knot. It contains one copy of Figure [6] as a 


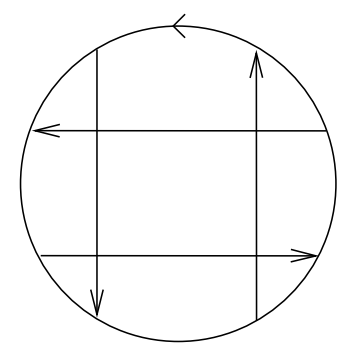

FiguRE 6 . The signed sum of subdiagrams of this form is invariant under $\Omega_{1}$-moves and $\Omega_{2}$-moves, but not $\Omega_{3}$-moves.

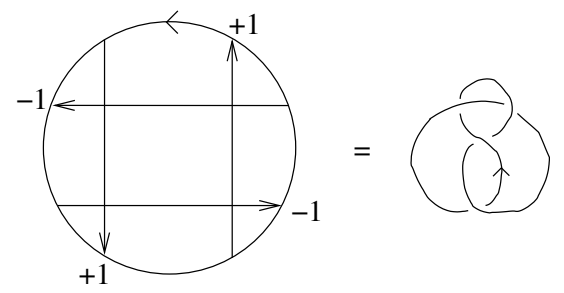

Figure 7. A Gauss diagram for a figure eight knot.
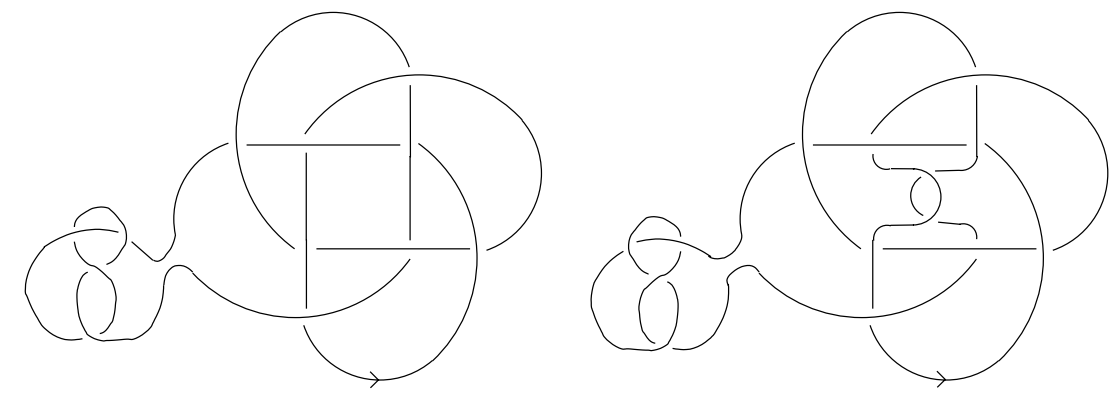

FiguRE 8 . These diagrams have different winding numbers. The pair is $\Omega_{1}$-dependent, $\Omega_{2}$-dependent, and $\Omega_{3}$-dependent.

subdiagram with sign $1^{2}(-1)^{2}=1$. Its mirror image has the same Gauss diagram with the arrows and signs (but not the orientation of $S^{1}$ ) reversed, and this does not contain any copies of Figure 6 as a subdiagram. Figure 7 and its mirror image have different winding numbers, so the pair is $\Omega_{1}$-dependent and $\Omega_{3}$-dependent.

Östlund's count is also additive under a connected sum of diagrams. Thus, if $\left(D, D^{\prime}\right)$ is an $\Omega_{2}$-dependent diagram pair constructed according to Theorem 2 , where $D$ and $D^{\prime}$ differ only by an $\Omega_{2}$-move, and $\left(E, E^{\prime}\right)$ is one of Östlund's $\Omega_{1}$ dependent, $\Omega_{3}$-dependent diagram pairs, then the diagrams $D \# E$ and $D^{\prime} \# E^{\prime}$ will form an $\Omega_{1}$-dependent, $\Omega_{2}$-dependent, $\Omega_{3}$-dependent diagram pair.

Figure 8 gives an example. 


\section{REFERENCES}

1. V. O. Manturov. Knot Theory. CRC Press, 2004. Appendix A. MR2068425

2. Olof-Petter Östlund. Invariants of knot diagrams and relations among Reidemeister moves. $J$. Knot Theory Ramifications, 10(8):1215-1227, 2001. MR.1871226 (2002j:57021)

3. K. Reidemeister. Knotten und gruppen. Abh. Math. Sem. Univ. Hamburg, 1927.

Department of Mathematics, Indiana University, Bloomington, Indiana 47405

E-mail address: thagge@indiana.edu 